\title{
Cerebrospinal fluid of chronic osteoarthritic patients induced interleukin- 6 release in human glial cell-line T98G
}

\section{Weiling Liu}

National University Health System

Chunmei Li

National University of Singapore

Francis Chee Kuan Tan

National University Health System

Hong Jye Neo

National University Health System

Yiong Huak Chan

National University of Singapore

Chian-Ming Low

National University of Singapore

Tat Leang Lee ( $\nabla$ analeetl@nus.edu.sg )

National University of Singapore https://orcid.org/0000-0003-4651-9222

Research article

Keywords: chronic pain, osteoarthritis, interleukin-6, cytokine

Posted Date: October 8th, 2019

DOl: https://doi.org/10.21203/rs.2.15747/v1

License: (c) (i) This work is licensed under a Creative Commons Attribution 4.0 International License.

Read Full License

Version of Record: A version of this preprint was published at BMC Anesthesiology on March 25th, 2020. See the published version at https://doi.org/10.1186/s12871-020-00985-0. 


\section{Abstract}

\section{Background:}

Chronic osteoarthritic pain is not well understood in terms of its pathophysiological mechanism. Activated glial cells are thought to play a role in the maintenance of chronic pain. T98G glioblastoma cell line was previously observed to release higher amounts of interleukin-6 (IL-6) when treated with cerebrospinal fluid (CSF) from patients with another chronic pain condition, post-herpetic neuralgia. In this study, we investigated the ability of CSF from patients diagnosed with knee osteoarthritis (OA) suffering from chronic pain, to trigger the release of pro-inflammatory cytokines, IL-6, IL-1 beta and tumour necrosis factor alpha (TNF-a) from T98G. Characterization of upstream signalling was also explored.

\section{Methods:}

$15 \mathrm{OA}$ patients undergoing total knee replacement and 15 patients without pain undergoing surgery with spinal anaesthesia were prospectively recruited. CSF was collected during anaesthesia prior to surgery. CSF were added to cultured T98G cells. IL-6, IL-1 $\beta$ and TNF- $\alpha$ release from T98G cells were measured using enzyme immunoassay. Antibody array and western blotting were performed on CSF-triggered T98G cell lysates to identify possible signalling targets. Age, gender and pain scores were recorded. MannWhitney $\mathrm{U}$ test was used to compare IL-6 release and protein expression between groups. Association between IL-6 and pain score was analysed using linear regression.

Results:

Compared to CSF of patients without pain, significantly higher IL-6 levels were released by T98G cells when induced by CSF from OA patients. The IL-6 levels showed positive association with pain score (adjusted B estimate $=10.1$ (95\% Confidence Interval 4.3-15.9); $p=0.001$ ). Array conducted with OA pain patient pooled CSF identified several proteins being upregulated greater than 2-fold including STE20related kinase adaptor protein and spleen tyrosine kinase. Further validation in western blotting of individual samples was unable to show similar significant increase.

Conclusion: 
IL-6 is a potential biomarker crucial in the maintenance phase of chronic pain in both OA and postherpetic neuralgia (PHN). Identification of signalling pathways involved in pain using pooled CSF on antibody array yield several protein of interest. However, those targets are inconsistent when individually validated using western blotting. Exploring other protein targets is required to identify the signalling mechanism of chronic pain.

\section{Background}

Osteoarthritis (OA) is a common joint degenerative disease affecting many patients worldwide. It is characterised by the progressive loss of articular cartilage, joint space narrowing, secondary bone hyperplasia and atrophy of joint muscles [1]. This disease affects weight-bearing joints and is more severe in the hips and knees. The hallmark symptom in OA frequently experienced by patients is localised chronic pain, which reduces their quality of life [2]. There is currently no cure for knee OA and the available treatments include use of medications, physical therapy or surgery that involves total joint replacement $[3,4]$. Despite having medications to alleviate pain, chronic pain is still a major problem for OA patients.

There is a lack of understanding about the signalling pathways involved in pain. Mechanisms underlying chronic pain may involve activation of a series of signalling molecules leading to increased peripheral and/or central sensitisation. Some studies evaluated inflammatory cytokines such as tumour necrosis

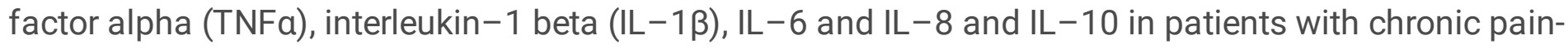
related diseases. For instance, IL -6 is elevated in cerebrospinal fluid (CSF) of patients with complex regional pain syndrome (CRPS) [5] and elevated IL -8 in patients with fibromyalgia [6].

Other studies showed chronic pain in OA being mediated by changes in neurotrophic factors, chemokines and interleukins as a response by chondrocytes or synovial cells at the joint in the synovial fluid [2]. Some showed glia activation related to pathogenesis of chronic pain [7]. Besides supporting neuronal cells, glial cells are found to have key roles in pain modulation. The exact role has not been elucidated [8].

Our group previously showed that CSF of post-herpetic neuralgia (PHN) chronic pain patients induced elevated IL -6 release from human glioblastoma T98G cells [9]. Therefore, we hypothesised that IL -6 is crucial in the maintenance phase of chronic pain signalling. In this study, we aimed to investigate whether CSF from a different chronic pain model (i.e. knee OA) could induce the release of proinflammatory cytokines IL $-6, I L-1 \beta$ and TNFa from T98G cells. We also investigate possible signalling pathways involved in chronic OA pain.

\section{Methods}

\section{Study design and patient population}

The procedure for CSF collection was approved by our Institutional Ethics Board and with written informed consent was obtained from each patient (DSRB Ref No. 2011/01710). Patients (both the pain 
group and the group without pain) were prospectively recruited by the attending anaesthesiologists prior to surgery. All patients recruited in the pain group satisfied the following inclusion criteria: (a) Confirmed OA of knees from history and x-ray of the knees by orthopaedic surgeons, (b) Numeric pain rating scale of more than 3 ( 0 being no pain; 10 being worst possible imaginable pain), (c) Knee pain of more than six months duration, (d) Scheduled for total knee replacement surgery under spinal anaesthesia, (e) Age of twenty-one years and above, $(f)$ No other concurrent chronic pain conditions. Patients without pain and undergoing surgery with spinal anaesthesia served as the control group. The inclusion criteria for no pain group include: (a) No history of chronic pain over the past one year; (b) Patient consented for surgery below the umbilicus under a spinal anaesthesia, (c) Age of twenty-one years and above. Pregnant patients or patients with severe end-organ function impairment (e.g. heart, lungs, liver and kidneys) were excluded.

CSF was obtained during the conduct of spinal anaesthesia, where two-ml of CSF was withdrawn after the dura was punctured, before injecting local anaesthetic into the subarachnoid space to effect the spinal anaesthesia. A total of 15 chronic knee OA pain patients and 15 no pain patients were recruited. CSF samples were then stored at $-80^{\circ} \mathrm{C}$. Patients' demographics such as gender and age were also recorded.

\section{Measurement of pro-inflammatory cytokine release in cell culture}

Human glioblastoma cell line T98G (ATCC-CRL1690, American Type Culture Collection, Manassas, VA) was cultured as previously described [9]. In brief, Eagle's Minimum Essential Medium (Gibco, Grand Island, New York) (EMEM) containing 10\% fetal bovine serum (FBS) (HyClone, Utah, USA) and 1X antibiotic-anti-mycotic cocktail (HyClone, Utah, USA) was used.

T98G cells were grown to approximately $90 \%$ confluency in 12-well plates. CSF from patients were filtered using 0.22 micron filter prior addition into cultured T98G cells. The culture medium was replaced with FBS-free EMEM medium before addition of $50 \mu \mathrm{l} \mathrm{CSF}$ along with $32 \mu \mathrm{g} / \mathrm{ml}$ lipopolysaccharide (LPS) from Escherichia coli 055:B5 (Sigma Aldrich, St Louis MO, USA) to the T98G cells. Cells were induced for 48 hours at $37^{\circ} \mathrm{C}$ and $5 \% \mathrm{CO}_{2}$ environment. LPS was used to enhance cytokine release from the cells $[9,10]$. After 48 hours, the medium containing "cytokine release" was collected and amount of IL-6 in this supernatant was measured in duplicates using enzyme-linked immunosorbent assay (ELISA) according to manufacturer's instructions (DY206, R\&D Systems, Minneapolis, USA). The detection range for the IL6 standard used was between $9.38 \mathrm{pg} / \mathrm{ml}$ to $600 \mathrm{pg} / \mathrm{ml}$.

Using supernatant from the above 48 hour CSF-triggered T98G protocol, TNFa and IL-1 $1 \beta$ levels were also determined with ELISA assays- Human TNFa Duoset ELISA (DY210) and Human IL-1ß/IL-1F2 Duoset (DY201) respectively (R\&D Systems, Minneapolis, USA). Detection range of standards for TNFa was 15.6 $\mathrm{pg} / \mathrm{ml}$ to $1000 \mathrm{pg} / \mathrm{ml}$; and IL-1 $\beta$ was $3.91 \mathrm{pg} / \mathrm{ml}$ to $250 \mathrm{pg} / \mathrm{ml}$. 


\section{Antibody array}

Nuclear factor kappa light chain enhancer of activated B cells (NFKB) phospho antibody array (PNK215, Fullmoon Biosystems, CA, USA) was used to screen for possible protein activity and protein expression profiling in our samples. This array applied an ELISA-based technique where samples were biotinylated before adding to the array slide containing affixed antibodies. Biotin on samples upon interaction with dye-labelled streptavidin on array would generate the fluorescence signal. Patient CSF-triggered T98G cells were harvested and lysed with CelLytic MT Cell Lysis Reagent (C3228; Sigma Aldrich). Total protein from T98G cell lysate were quantified using bicinchoninic acid (BCA) assay (Pierce BCA Protein Assay Kit, Thermo Scientific, USA). Pooled OA pain sample and no-pain sample $(n=6)$ was added to each antibody array slide and assay was performed according to manufacturer's instructions. Array image was captured using array scanner (GenePix 4000B; Molecular Probes, CA, USA). Analysis of array data was done using Genescan software. Comparison of signals between OA pain pooled sample and no pain pooled sample was done after normalization with glyceraldehyde-3-phosphate dehydrogenase (GAPDH).

\section{Western Blot analysis}

To validate the fold increase as observed in the antibody array for OA triggered T98G cells, all individual OA and no pain-CSF triggered T98G cell lysates $(n=15$ each) were checked for protein expression level of targets with Western Blotting. Ten micrograms of each CSF-triggered T98G cell lysates were loaded per lane of $10 \%$ Tris-glycine gels and run using Sodium dodecyl sulphate polyacrylamide gel electrophoresis (SDS-PAGE). Proteins on gel were transferred onto Polyvinylidene difluoride (PVDF) membrane. 0.1\% Tween-20 added into Tris-buffered saline (TBS-T) was used to wash membranes and prepare antibodies. Non-specific binding sites on the membranes were blocked in $5 \% \mathrm{w} / \mathrm{v}$ Bovine serum albumin (BSA)-TBS-T or milk-TBS-T. BSA was used when checking for spleen tyrosine kinase (Syk); while milk was used for STE20-related kinase adaptor protein (STRAD) and GAPDH. Primary antibodies used in this study are: STRAD (G-8) (Santa Cruz Biotechnology, CA, USA); Syk (D3Z1E) (Cell Signaling Technology Inc, MA, USA); and GAPDH (MAB374) (EMD Millipore, Darmstadt, Germany). GAPDH, with a molecular weight of $37 \mathrm{kDa}$, was used as the loading control for normalization. Membranes were imaged with ChemiDoc XRS+ system. Densitometry quantification was done with ImageLab software.

\section{Statistical Analysis}

All data were analysed using GraphPad Prism v5.0 (GraphPad, La Jolla, CA, USA) and SPSS v24.0 (IBM SPSS, Armonk NY, USA). Cytokine levels was checked for normality and deemed not normally distributed, thus non-parametric Mann Whitney-U test was used. Mann-Whitney $U$ test was also used to analyse STRAD and Syk protein levels from western blotting between groups. Simple and multivariate linear regression model analyses were used to study the relationship of pain score and cytokine level. A $p$-value of less than 0.05 was considered statistically significant. 


\section{Results}

\section{Patient demographics}

The average age of patients in our cohort is $61 \pm 13$ years. Demographics of OA pain and no pain patients are described in Table 1. The control group are patients without pain.

\section{IL-6 release from T98G cells}

The IL- 6 levels in the CSF samples from OA and no pain patients were below the lowest detection limit of the ELISA kit $(9.38 \mathrm{pg} / \mathrm{ml})$ used. In order to detect IL -6 levels using the ELISA method, the additional induction methodology used in [9] were used to amplify the IL -6 changes.

In the absence of CSF, addition of LPS to T98G cells did not trigger detectable levels of IL-6 release [9]. When patients' CSF and LPS were added to T98G cells, IL -6 release was detected in the cell culture media. $67.0 \pm 17.7 \mathrm{pg} / \mathrm{ml}$ of IL -6 was detected when induced with OA CSF $(n=15)$ whereas $23.7 \pm 3.4$ $\mathrm{pg} / \mathrm{ml}$ of IL -6 was detected for no pain CSF group $(\mathrm{n}=15)$. There was a statistically significant higher amount of IL -6 released from T98G cells triggered with OA CSF as compared to no pain CSF (Figure 1; Mann Whitney-U test, $p=0.002$ ).

\section{TNFa and IL $-1 \beta$ release from T98G cells}

TNFa and IL-1 $\beta$ (lowest detection range of $15.6 \mathrm{pg} / \mathrm{ml}$ and $3.91 \mathrm{pg} / \mathrm{ml}$ respectively), were evaluated and found to be undetectable in T98G cell culture media when induced with CSF (OA/no pain) and LPS, with their respective ELISA kits.

\section{Relationship between IL -6 release and pain score}

There was a significant positive relationship between pain score and IL-6 cytokine levels (unadjusted B estimate $=7.1(95 \%$ Confidence Interval $($ C. I. $)=2.1-12.1) ; p=0.007)$. After adjusting for age and gender in the multivariate model, the significant association persists (adjusted $\mathrm{B}$ estimate $=10.1(95 \% \mathrm{Cl}=4.3-$ 15.9); $p=0.001)$.

\section{Possible targets found in antibody array}

The antibody array used consisted of 6 replicates of 215 antibodies for targets related to the NFKB pathway. $6 \mathrm{CSF}$ samples of $40 \mu \mathrm{g}$ each were pooled together to give a total $240 \mu \mathrm{g}$ protein for the array testing. The pooled OA-CSF triggered T98G cell lysate of $240 \mu \mathrm{g}$ was added to one array slide; while pooled no pain-CSF triggered T98G cell lysate was added to another slide. The fluorescence signal of all 
6 replicates were recorded and the average was calculated. GAPDH was used to normalise the signal for both OA and no pain samples. Those with a higher fold increase in OA-CSF triggered T98G cell lysate as compared to that of no pain CSF were noted. The more notable ones having greater than 3 -fold increase were STRAD ( 4.25 fold), Syk (3.40 fold), lymphocyte-specific protein tyrosine kinase (3.30 fold) and IkappaB-alpha (3.07 fold) (Figure 2). STRAD and Syk were chosen for further validation using western blotting.

\section{Protein expression of STRAD and Syk}

The molecular weight of STRAD is $48 \mathrm{kDa}$. For STRAD protein, there is a higher protein expression level in OA patients $(1.26 \pm 0.54)$ as compared to patients without pain $(1.05 \pm 0.42)$. However, the difference was not statistically significant $(p=0.298)$ (Figure 3$)$.

Syk has a molecular weight of $72 \mathrm{kDa}$. Similarly, higher protein expression level was observed in OA patients $(2.98 \pm 2.9)$ as compared to no pain patients $(1.96 \pm 1.4)$ for Syk protein. This higher protein expression in OA patients was not statistically significant $(p=0.183)$ (Figure 4$)$.

\section{Discussion}

CSF from OA patients was able to stimulate an increase in IL -6 release from cultured T98G cells as compared to the stimulation using CSF from patients free from pain. This trend is consistent with our previous work published on a different chronic pain cohort (PHN patients) [9]. Using the same method to measure IL -6 in both $\mathrm{OA}$ and PHN [9], this suggests that the methodology of measuring induced release of IL- 6 in T98G using patient CSF in conjunction with LPS allows the IL- 6 to be used as a pain biomarker. The positive correlation between the patient pain scores and the IL -6 release levels measured further shows that the method works for both OA and PHN; and possibly in other chronic pain $[5,13,14$, $15,16,17]$. CSFs from patients from two different chronic pain models (OA and PHN) significantly increase IL -6 release from T98G cells, suggesting that the CSF from patients with chronic pain contain triggering factor(s). IL -6 is a multi-functional protein cytokine known to play a role in the modulation of signalling in the central nervous system (CNS) such as those influencing immune responses, inflammation and wounds [11]. IL -6 has been implicated in various diseases related to pathological pain. Elevated IL -6 was observed in other pain-related studies such as CRPS or chronic low back pain that used CSF or blood serum samples $[5,12,13,14,15,16,17]$.

In our current and previous studies, we found that CSF in patients had undetectable levels of IL-6, irrespective of their pain score (no pain, $\mathrm{OA}$ and PHN). Hence, possible confounding effect of baseline IL6 is minimised. Our data also suggest that IL- 6 in CSF exert minimum central sensitisation as IL-6 is present at undetectable levels in the CSF. The present work also showed patients with higher pain score ratings had significantly higher level of IL-6 released by T98G cells when stimulated with their CSFs. Cytokines like IL -6 release are increased in activated glial cells particularly microglia and astrocytes in relation to pain [18]. Some studies that analysed serum or plasma reported direct IL-6 detection and 
showed significant correlation between IL-6 and pain rating in chemotherapy induced peripheral neuropathy and chronic lower back pain $[12,13]$. This suggests a possible role of IL-6 in peripheral sensitisation.

When more IL-6 release from T98G cells was observed in the trigger using CSF from OA patients, the signalling pathway upstream of IL- 6 was investigated. NFKB explorer array was chosen as it is one of the common upstream molecular players of $\mathrm{IL}-6$. Several protein targets were found to be elevated with greater than two-fold in OA CSF triggered T98G cells as compared to no pain CSF triggered ones (Figure 2). With protein expression greater in OA pain samples, these targets could be important in delineating the chronic pain signalling mechanism. Two protein targets showing highest fold increase of 4.2 and 3.4 fold in OA CSF in the array are STRAD and Syk respectively. STRAD, predominantly found in CNS, is known to complex with serine/threonine kinase LKB1 and scaffold protein MO25a to activate AMP-activated protein kinase (AMPK) signalling pathway which in turn influence various downstream effects [19]. NFKB and IL -6 are downstream of AMPK [20]. Syk plays a role in signal transduction influencing cells of the immune system- both B and T cells. Syk leads to downstream release of pro-inflammatory cytokines [21]. When they were further validated in western blotting, STRAD and Syk protein showed the same trend. Even though they had higher expression in T98G cell lysate triggered by OA CSF, the difference was not evident enough to confirm that they are key players in chronic pain signalling. Increasing sample size and altering time point of T98G lysate collection may be required to validate the importance of STRAD and Syk in pain signalling. Further exploration of other identified potential protein targets may also be useful in delineating a possible signalling pathway that OA plays in T98G cells.

The strength of our study is the use of human CSF to elucidate possible biomarkers of chronic pain. CSF, as compared to peripheral blood, has a more direct contact with the CNS environment. Finding pain biomarkers such as IL -6 will allow a more objective measurement of pain as compared to patient reported VAS pain scores. Our study shows significant and positive correlation of the IL- 6 and the reported pain scores from patients. Using high IL-6 release as an indication of the activation of the pain pathways may prove to be a better indicator than perceived pain reported by patients. That will allow the physicians to respond with the correct treatment to manage the pain.

The limitation was the failure to conclusively isolate the signalling pathway that leads to IL-6 release using the antibody array and western blotting. In the attempt to obtain highest number of targets from the array, we used T98G induced with 6 pooled CSF of OA patient with the highest pain score to compare it with patient CSF without pain. This is to prevent T98G samples treated with lower pain score CSF to confound the results, as patient pain scores may be perceived to be higher person to person. However, on retrospect, this may contribute to higher false positive targets when we conducted western blotting on all the samples. 48-hour treatment time point may also not be the peak expression of these targets, leading to overall lack of significance in western blotting results. Another limitation was that only a limited number of antibodies were tested.

\section{Conclusion}


In summary, CSF from chronic OA patients was able to induce higher levels of IL-6 release from T98G cells, hence showing a similar trend as our previous study in a different chronic pain model. This common phenomenon suggests the presence of yet-to-be identified factor(s) in CSF of patients with chronic pain which could potentially serve as clinically informative biomarker(s). Further studies are needed to investigate the relevance of IL -6 release by T98G glial cells in other chronic pain models, and this in vitro method as an objective assessment of pain severity. Even though potential upstream players STRAD and Syk were not validated as players in the chronic pain signalling, other targets with greater than 2-fold increase in OA sample detected in the antibody array can be probed further to identify possible players in the signalling mechanism of chronic pain.

\section{Abbreviations}

AMPK: AMP-activated protein kinase; ATCC: American Type Culture Collection; BCA: bicinchoninic acid; BSA: Bovine serum albumin; Cl: Confidence Interval; CNS: Central Nervous System, CRPS: Complex Regional Pain Syndrome, CSF: Cerebrospinal fluid; ELISA: Enzyme-linked immunosorbent assay; EMEM: Eagle's minimum essential medium; F: female; FBS: Fetal Bovine Serum; GAPDH: glyceraldehyde-3phosphate dehydrogenase; IL: Interleukin; LPS: Lipopolysaccharide; M: male; NFkB: Nuclear factor kappa light chain enhancer of activated B cells; OA: Osteoarthritis; PHN: Post-herpetic Neuralgia; SD: standard deviation; SDS-PAGE: Sodium dodecyl sulphate polyacrylamide gel electrophoresis; STRAD: STE20related kinase adaptor protein; Syk: Spleen tyrosine kinase; TNFa: Tumour Necrosis Factor-alpha

\section{Declarations}

\section{Acknowledgements}

Not applicable.

\section{Funding}

This work was supported by National University Health System Bench to Bedside Grant (R-184-000232-515) and the Department of Anaesthesia, National University of Singapore.

\section{Availability of data and materials}

The datasets generated and analysed during the current study are available from the corresponding author on reasonable request.

\section{Authors' contributions}


WL helped with conducting of study, data analysis and writing of manuscript. CL helped with conducting of study and preparation of manuscript. FCKT helped with data analysis and preparation of manuscript. HJN helped with collecting CSF samples and preparation of manuscript. YHC helped with data interpretation and preparation of manuscript. CML helped with study design and implementation, data interpretation and preparation of manuscript. TLL helped with study design and implementation, collecting CSF samples, data interpretation and preparation of manuscript. All authors approved the final manuscript.

\section{Ethics approval and consent to participate}

All experimental procedures were approved by our Institutional Ethics Board and written informed consent were obtained from all recruited patients (DSRB Ref No. 2011/01710).

\section{Competing Interests}

The authors declare that they have no competing interests.

\section{References}

1. DRAY A, READ SJ. Arthritis and pain. Future targets to control osteoarthritis pain. Arthritis Research \& Therapy. 2007;9(3):212.

2. MILLER RE, MILLER RJ, MALFAIT A-M. Osteoarthritis joint pain: The cytokine connection. Cytokine. 2014;70(2):185-193.

3. Singapore Burden of Disease Study 2010. Ministry of Health Singapore. https://www.moh.gov.sg/docs/librariesprovider5/resources-statistics/reports/singapore-burden-ofdisease-study-2010-report_v3.pdf. Accessed April 22, 2019.

4. MUSHTAQ S, CHOUDHARY R, SCANZELLO CR. Non-surgical treatment of osteoarthritis-related pain in the elderly. Current Reviews in Musculoskeletal Medicine. 2011;4(3):113-122.

5. ALEXANDER GM, RIJN MAV, HILTEN JJV, PERREAULT MJ, SCHWARTZMAN RJ. Changes in cerebrospinal fluid levels of pro-inflammatory cytokines in CRPS. Pain. 2005;116(3):213-219.

6. BJURSTROM MF, GIRON SE, GRIFFIS CA. Cerebrospinal Fluid Cytokines and Neurotrophic Factors in Human Chronic Pain Populations: A Comprehensive Review. Pain Practice. 2014;16(2):183-203.

7. LOGGIA ML, CHONDE DB, AKEJU O, et al. Evidence for brain glial activation in chronic pain patients. Brain. 2015;138(3):604-615.

8. SHERIDAN C. Glial cells on the radar. Nature Biotechnology. 2009;27(2):114-116.

9. TAY AS, LIU EH, LEE TL, et al. Cerebrospinal fluid of postherpetic neuralgia patients induced interleukin-6 release in human glial cell-line T98G. Neurochemistry International. 2013;63(5):517521. 
10. GRIMALDI M, NAVARRA P, POZZOLI G, PREZIOSI P, SCHETTINI G. Bacterial Lipopolysaccharide Increases Interleukin- 6 and Prostaglandin Release in Rat Cortical Type I Astrocytes by Different Mechanisms: Role of Anti-inflammatory Agents. Biochemical and Biophysical Research Communications. 1998;250(3):798-804.

11. CHOY E., ROSE-JOHN S. Interleukin- 6 as a multifunctional regulator: inflammation, immune response, and fibrosis. Journal of Scleroderma and Related Disorders. 2017: S1-S5.

12. STARKWEATHER A. Increased Interleukin-6 Activity Associated with Painful Chemotherapy-Induced Peripheral Neuropathy in Women after Breast Cancer Treatment. Nursing Research and Practice. 2010;2010:1-9.

13. HEFFNER KL, FRANCE CR, TROST Z, NG HM, PIGEON WR. Chronic low back pain, sleep disturbance, and interleukin-6. The Clinical journal of pain. 2011;27(1):35.

14. KRAYCHETE DC, SAKATA RK, ISSY AM, BACELLAR O, SANTOS-JESUS R, CARVALHO EM. Serum cytokine levels in patients with chronic low back pain due to herniated disc: analytical crosssectional study. Sao Paulo Medical Journal. 128(5) 2010:259-262.

15. WEBER KT, ALIPUI DO, SISON CP, et al. Serum levels of the proinflammatory cytokine interleukin- 6 vary based on diagnoses in individuals with lumbar intervertebral disc diseases. Arthritis research \& therapy. 18(1) 2016: 3.

16. PEDERSEN LM, SCHISTAD E, JACOBSEN LM, ROE C, GJERSTAD J. Serum levels of the proinflammatory interleukins $6(\mathrm{IL}-6)$ and $-8(\mathrm{IL}-8)$ in patients with lumbar radicular pain due to disc herniation: a 12-month prospective study. Brain, behavior, and immunity. 46 2015: 132-136.

17. ZHOU, YQ, LIU, Z, Liu, ZH, et al. Interleukin-6: an emerging regulator of pathological pain. Journal of neuroinflammation. 13(1) 2016:141.

18. WATKINS, LR, MILLIGAN, ED, \& MAIER, SF. Glial activation: a driving force for pathological pain. Trends in neurosciences. 24(8) 2001:450-455.

19. HAWLEY SA, BOUDEAU J, REID JL, et al. Complexes between the LKB1 tumor suppressor, STRADa/ $\beta$ and $\mathrm{MO} 25 \mathrm{a} / \beta$ are upstream kinases in the AMP-activated protein kinase cascade. Journal of biology. 2(4) 2003: 28.

20. SALMINEN, A, HYTTINEN, JM, \& KAARNIRANTA, K. AMP-activated protein kinase inhibits NF-KB signaling and inflammation: impact on healthspan and lifespan. Journal of molecular medicine. 89(7) 2011:667-676.

21. KYTTARIS, VC. The role of Syk in osteoarthritis. Clinical immunology. 144(3) 2012: 283.

\section{Table 1}

Table 1. Patient demographics. 


\begin{tabular}{l|ccc}
\hline Group & OA pain $(\mathrm{n}=15)$ & No pain $(\mathrm{n}=15)$ & $p$-value \\
\hline Age (years) & $60 \pm 13$ & $63 \pm 14$ & 0.618 \\
Gender & 7 male, 8 female & 14 male, 1 female & 0.014 \\
Pain Score & $7 \pm 2$ & 0 & $<0.001$ \\
History of diabetes mellitus, yes & $3(20 \%)$ & $5(33.3 \%)$ & 0.682 \\
History of hypertension, yes & $8(53.3 \%)$ & $8(53.3 \%)$ & 1 \\
History of peripheral vascular disease, yes & 0 & & \\
& & & \\
History of hyperlipidaemia, yes & & & \\
\end{tabular}

Data are presented as Mean \pm standard deviation (SD) or number (percentage). $p$-value of 0.05 is considered statistically significant.

\section{Figures}




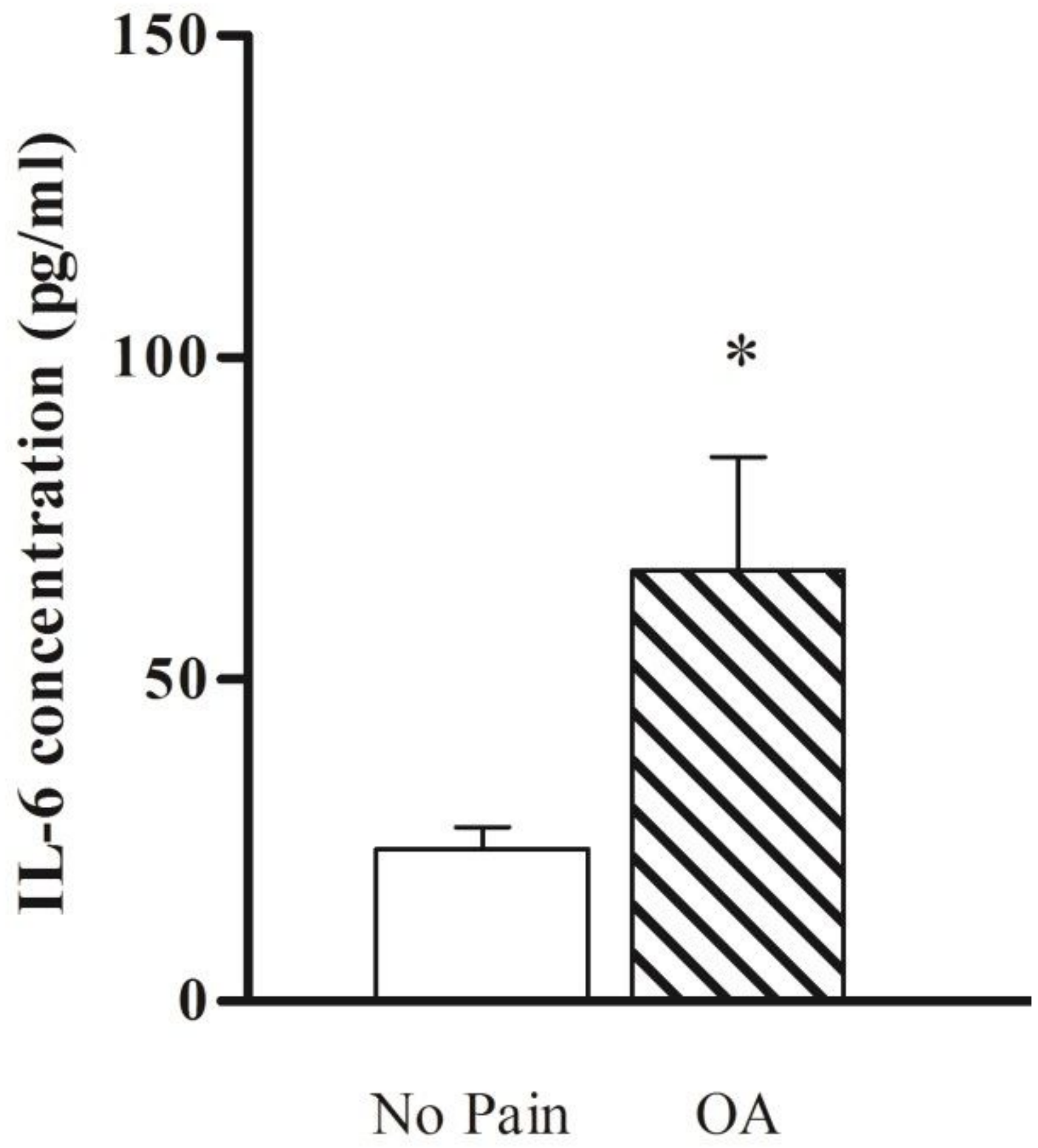

Figure 1

IL-6 release from no pain or OA pain CSF stimulated T98G cells. IL-6 release from T98G cells when triggered with $50 \mu \mathrm{l}$ CSF of either OA CSF or no pain CSF, in the presence of LPS. Bars are plotted as mean \pm standard error of mean (15 patients per group), ${ }^{\star} p<0.05$ when using Mann Whitney-U test. 


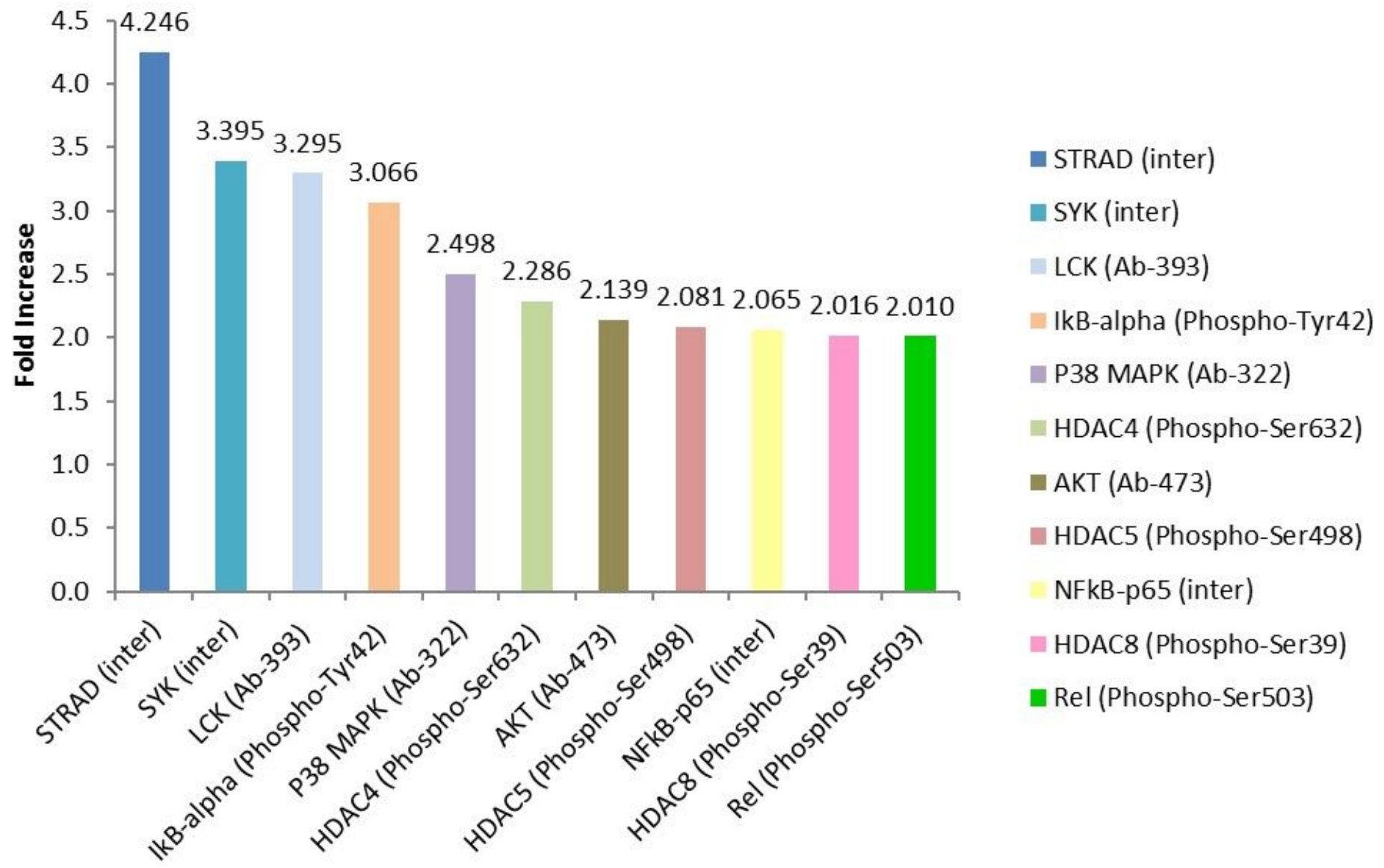

Figure 2

Graph of antibody protein targets greater than 2-fold increase in OA pain patients' pooled sample.

STRAD Protein Expression Level

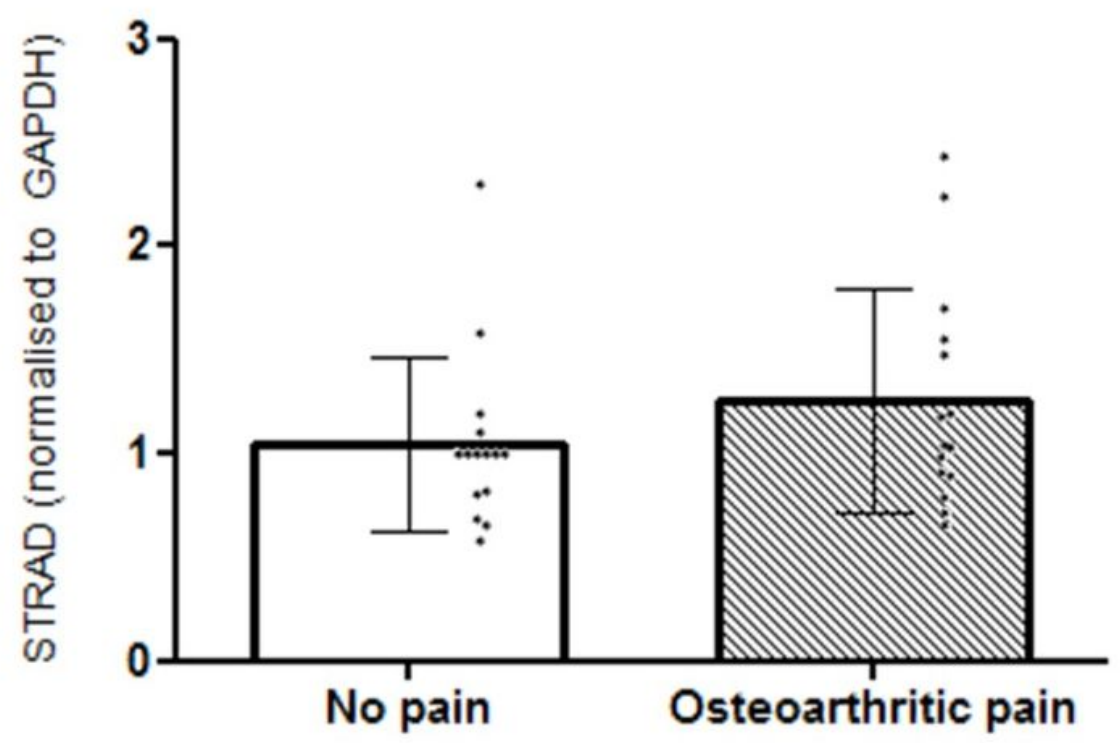

$\square$ No pain

Osteoarthritic pain

$n=15$

STRAD

GAPDH

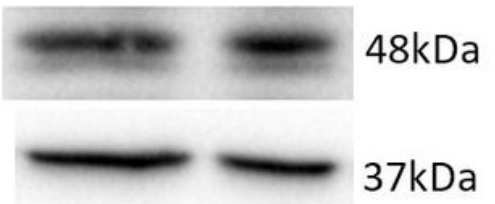

No pain OA

Figure 3 
STRAD protein expression level in western blotting. Each dot represents each individual patient STRAD expression in the T98G cell lysate. Bars are plotted as mean \pm SD (15 patients per group), ${ }^{\star} p<0.05$ when using Mann Whitney-U test.

\section{Syk Protein Expression Level}

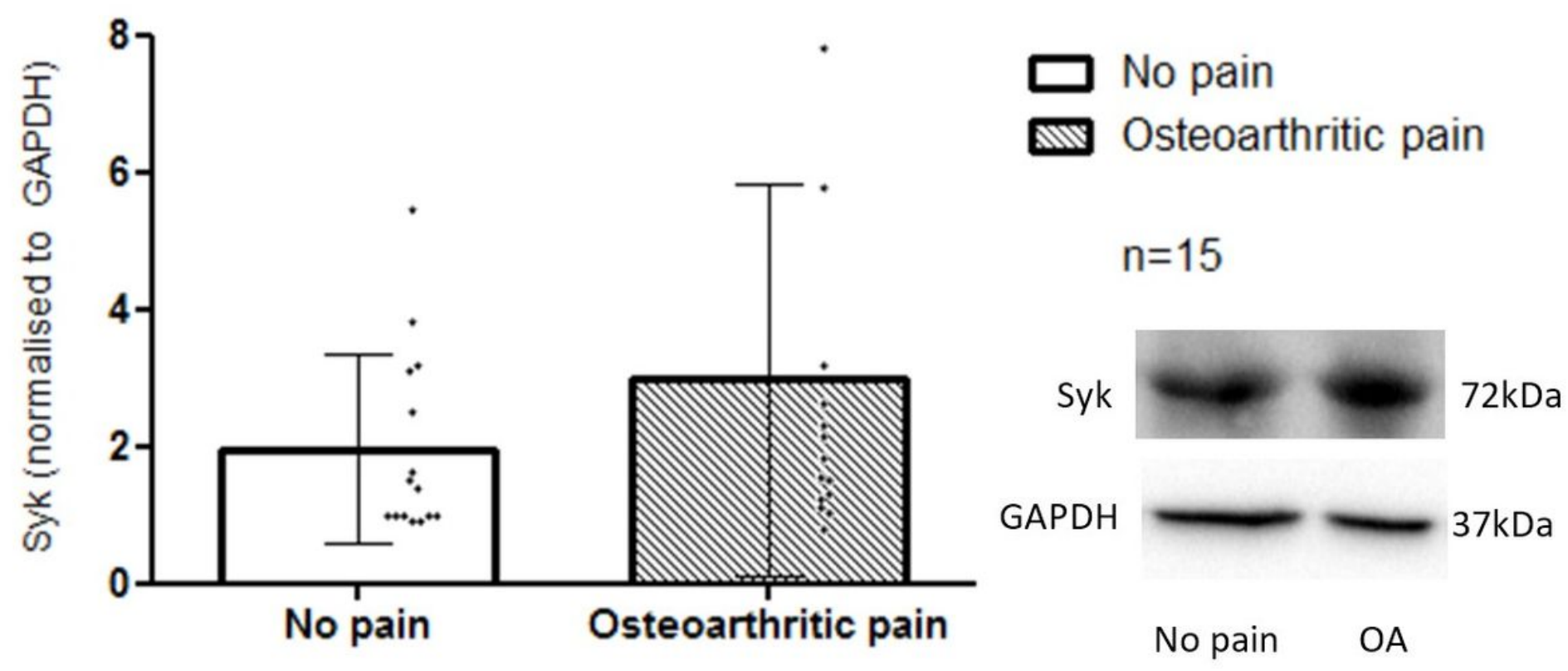

Figure 4

Syk protein expression level in western blotting. Each dot represents each individual patient STRAD expression in the T98G cell lysate. Bars are plotted as mean \pm SD (15 patients per group), ${ }^{\star} p<0.05$ when using Mann Whitney-U test. 